\title{
Combining Non-Rigid Registration with Non-Local Principle Component Analysis for Atomic Resolution EDS Mapping
}

Chenyu Zhang ${ }^{1}$, Albert $\mathrm{Oh}^{2}$, Andrew Yankovich ${ }^{3}$, Thomas Slater ${ }^{4}, \mathrm{Sarah} \mathrm{Haigh}^{4}$, Rebecca Willett $^{2}$, Paul M. Voyles ${ }^{1}$

1. Department of Materials Science and Engineering, University of Wisconsin-Madison, Madison, WI, 53706, USA

2. Department of Electrical and Computer Engineering, University of Wisconsin-Madison, Madison, WI, 53706, USA

3. Department of Physics, Chalmers University of Technology, Gothenburg, Sweden

${ }^{4}$ School of Materials, University of Manchester, Manchester, M13 9PL, UK.

Energy-dispersive x-ray Spectroscopy (EDS) spectrum imaging in the STEM can be used to create compositionally-sensitive images of materials at atomic resolution $[1,2]$. However, the $\mathrm{x}$ ray signal is very weak, so EDS spectrum image data sets are often dominated by Poisson noise. We have applied non-local principle component analysis (NLPCA) [3] to reduce the level of Poisson noise in a model atomic-resolution EDS spectrum image. The processed data has better contrast and lower noise compared to normal methods, enabling more clear visualization of fine spatial features.

EDS spectrum images with simultaneous HAADF images were acquired from Ca-stabilized $\mathrm{NdTiO}_{3}$ perovskite with $\mathrm{Ca}$ and $\mathrm{Nd}$ on the A sites. 100 spectrum image frames were acquired with $256 \times 256$ spatial pixels and 2048 energy channels. We applied non-rigid registration (NRR) [4] to the simultaneous HAADF images, and then implemented the shifts to each energy channel of each spectrum image. The resulting registered spectrum images were summed within seven characteristic X-ray lines and result in seven integrated elemental maps.

The NLPCA algorithm applies a non-local method for denoising hyperspectral data. It first performs patchization on the original data cube to get a collection of small noisy patches and divides the patches into clusters using k-means clustering. Poisson principle component analysis (PCA) is applied to each cluster to reduce Poisson noise. The final denoised image is reconstructed from the denoised clusters.

NLPCA method was applied to the seven integrated band elemental maps to reduce Poisson noise. The final denoised images show atomic structure in all bands except the $\mathrm{O} \mathrm{K} \alpha$ band, as shown in Figure 1. The NLPCA results clearly indicate that $\mathrm{Ca}$ atoms occupy $\mathrm{Nd}$ sites, which is an observation that is not visible before NLPCA but does agree with the known structure.

The reliability of the NLPCA algorithm and the best denoising parameters were studied with phantom EDS spectrum image data based on the experimental data but without noise. Three different $\mathrm{Ca}$ distributions were considered in the phantom data: a uniform distribution, $\mathrm{Ca}$ nanoparticles, and a random distribution. Poisson noise was added to the synthetic data, then denoised using NLPCA. The peak signal-noise ratio (PSNR) was used to measure the denoised image quality and was used to determine the most efficient set of NLPCA parameters. The results, shown in Figure 2, demonstrate that NLPCA is capable of reconstructing the phantom with Poisson noise corrupted data for all seven bands in three different Ca distributions [5]. 
References:

[1] M.-W. Chu, et al., Phys. Rev. Lett. 104, 196101 (2010).

[2] A. J. D’Alfonso, Phys. Rev. B 81, 100101 (2010).

[3] J. Salmon, et al, Journal of mathematical imaging and vision, 48 (2014), p.279-294.

[4] A.B. Yankovich, et al, Nat. Commun. 5 (2014), p.4155

[5] This work was supported by the US Department of Energy, Basic Energy Sciences, under grant DE-FG02-08ER46547.

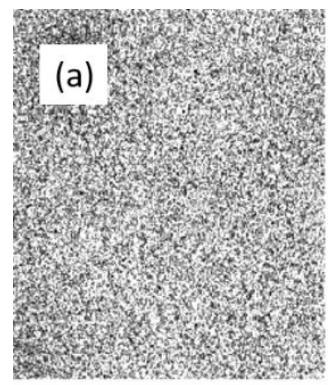

\section{(f)}
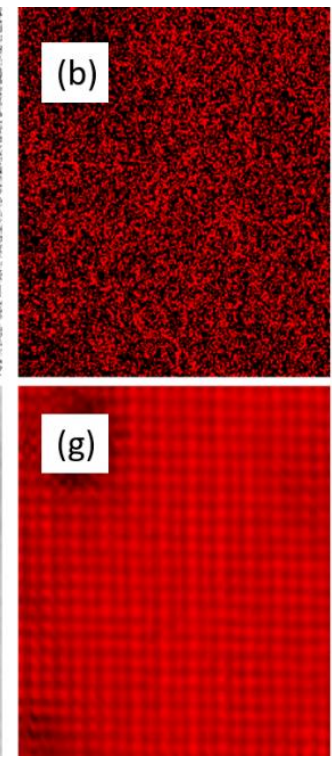
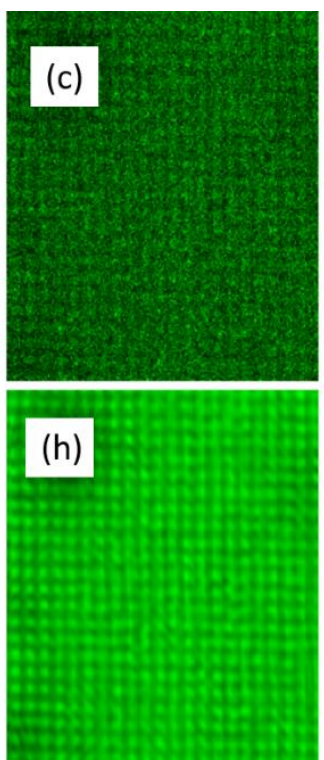
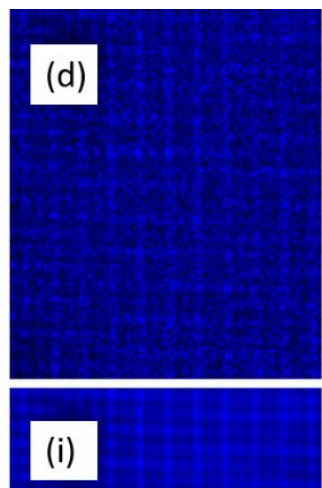
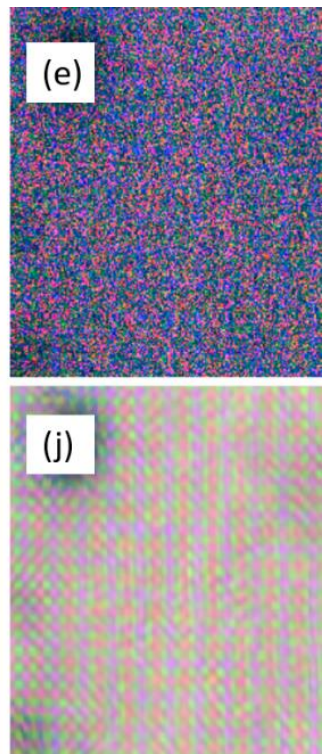

Figure 1 Experimental EDS elemental maps for (a) O (b) Ca (c) Ti and (d) Nd with overlaid image (e), (f)-(i) are the corresponding denoised maps with the overlaid image (j)
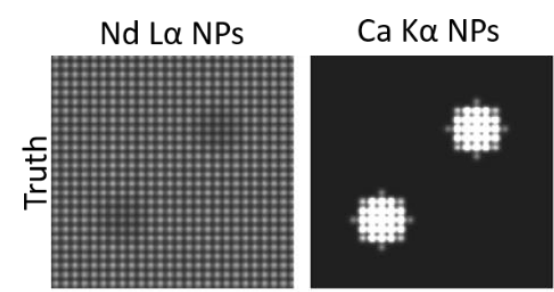

Ca Ka Random

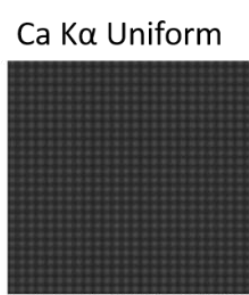

$\mathrm{O} \mathrm{K} \alpha$ Uniform
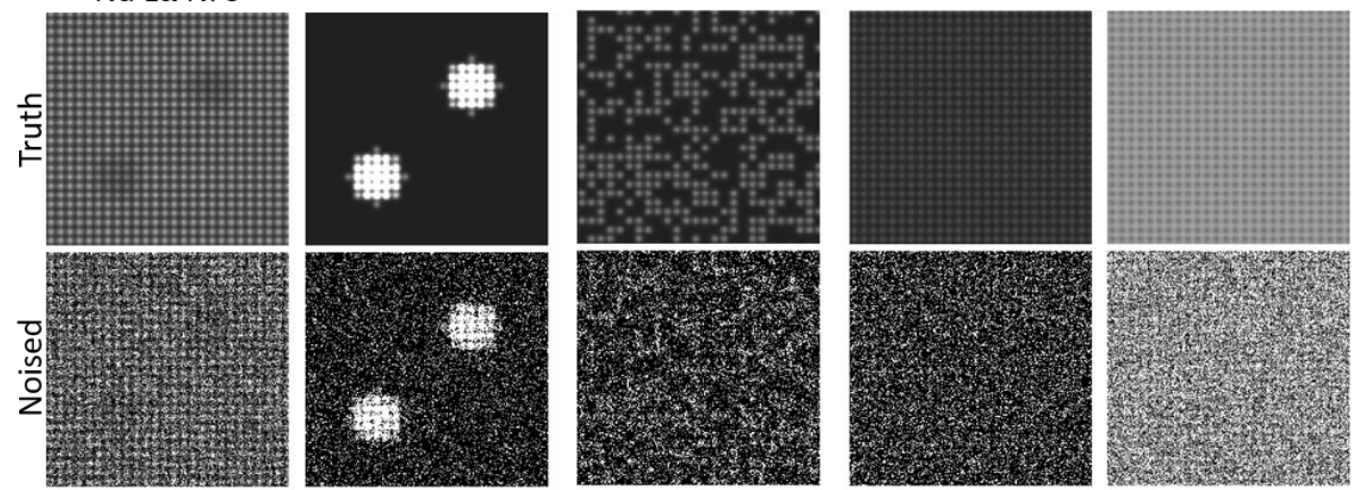

Ti Ka Uniform
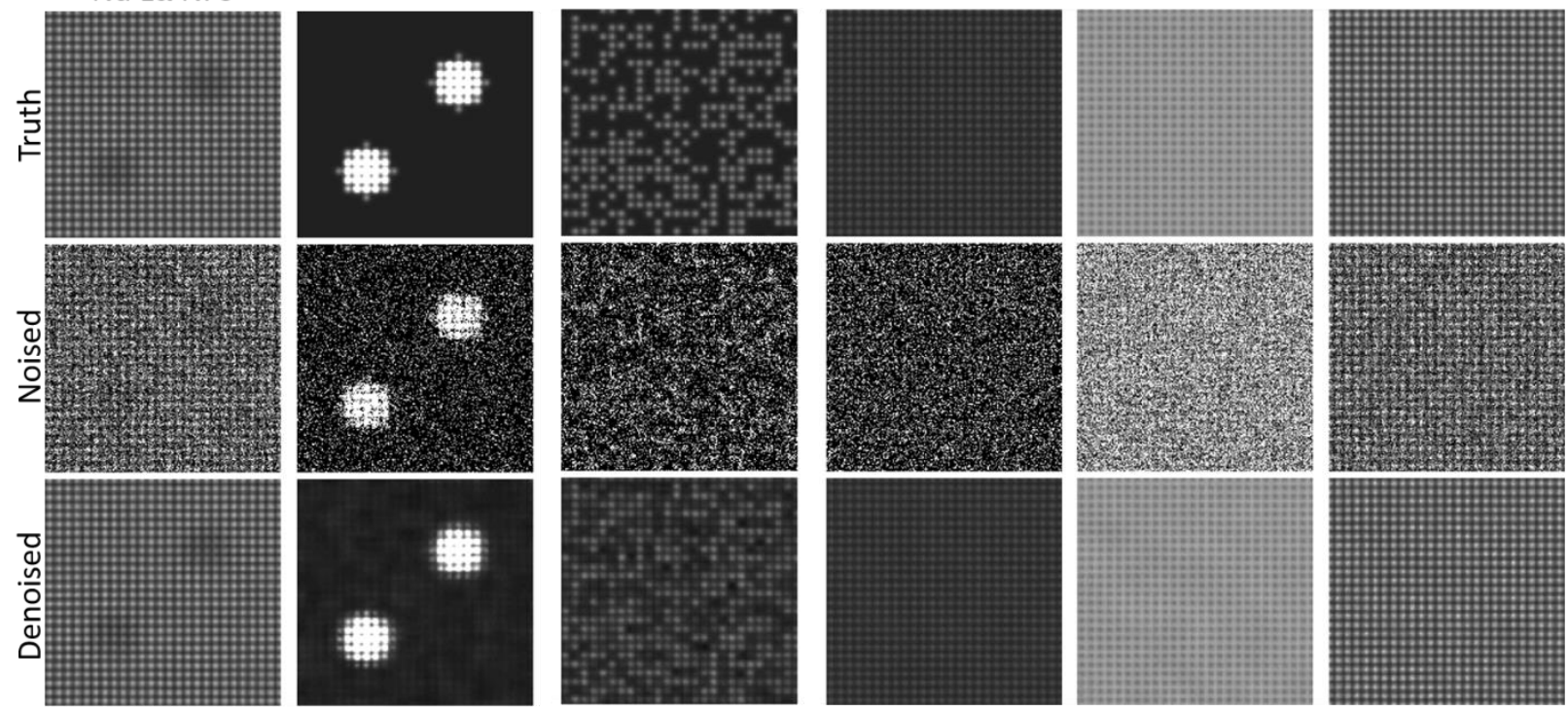

Figure 2 Phantom truth for $\mathrm{Nd} \mathrm{L} \alpha, \mathrm{Ca} \mathrm{K} \alpha, \mathrm{O} \mathrm{K} \alpha$ and $\mathrm{Ti} \mathrm{K} \alpha$ together with their noised image and the denoised result produced by NLPCA algorithm 